\title{
Modelo para predição de morbi-mortalidade e de necessidade de UTI pós-operatória em pacientes cirúrgicos oncológicos
}

\author{
Model for predicting morbidity and mortality and need for ICU post- \\ operative surgical patients with cancer
}

Tatiana Barboza Kronemberger ${ }^{1}$, José Otávio Costa Auler Jr²

Kronemberger TB, Auler Jr JOC. Modelo para predição de morbi-mortalidade e de necessidade de UTI pós-operatória em pacientes cirúrgicos oncológicos / Model for predicting morbidity and mortality and need for ICU postoperative surgical patients with cancer. Rev Med (São Paulo). 2013;92(1):43-51.

RESUMO: Vários escores de predição de morbi-mortalidade de pacientes submetidos a cirurgias, com possível necessidade de utilizarem leitos de UTI, vêm sendo testados. Atualmente a indicação de suporte intensivo pós-operatório para cirurgias eletivas é estabelecida de maneira subjetiva, podendo inclusive comprometer o desempenho do hospital levando à suspensão de cirurgias por falta de vagas de UTI. O escore mais utilizado foi proposto pela Sociedade Americana de Anestesiologia, sendo o ASA, como preditor de risco, porém, esta classificação está sujeita a variabilidade de interpretação. Outra proposta é a utilização do POSSUM que leva em conta doze variáveis fisiológicas e seis variáveis cirúrgicas. Também pode ser mencionada a escala de Karnofsky que classifica os pacientes de acordo com o grau de deficiências funcionais ocasionadas pela enfermidade que os acomete. O objetivo deste estudo foi correlacionar tais índices na busca de um melhor preditor para utilização de UTI e também verificar se as variáveis fisiológicas do POSSUM também poderiam ser utilizadas para compor uma melhor avaliação acerca da real necessidade de reserva desses leitos, a partir da análise dos prontuários de 186 pacientes submetidos a cirurgias oncológicas. Como resultados, podemos inferir que as variáveis fisiológicas que compõem o POSSUM são melhores preditores que os outros índices analisados para a reserva do leito de UTI. Também notamos que as variáveis idade, nível sérico de hemoglobina e a medida da pressão arterial sistólica seriam suficientes para predizer a real necessidade da utilização da vaga na unidade de terapia intensiva.

DESCRITORES: Oncologia; Neoplasias/cirurgia; Terapia intensiva; Medição de risco; Cirurgia geral; Fatores de risco; Unidades de terapia intensiva.

\begin{abstract}
Several scores for predicting morbidity and mortality in patients undergoing surgery, who will possibly use ICU beds, are being tested. Currently, the indication of intensive postoperative support for elective surgeries is established in a subjective way and may even endanger the performance of the hospital since it could spawn the suspension of operations due to lack of vacancies in the ICU. The most used score was proposed by the American Society of Anesthesiologists, ASA, as a risk predictor, however this classification is frequently submitted to different interpretations. It is also considered the use of POSSUM, which takes into account twelve physiological variables and six surgical variables. We could mention the Karnofsky scale as well, which classify patients according to the degree of functional disability caused by a neoplastic disease. The objective of this study was to correlate these elements in order to find a better predictor for ICU. Secondly, we intended to verify whether POSSUM physiological variables could be used as predictors to ICU reservation in surgeries. We reviewed 186 patients medical records undergoing oncologic surgeries. As a result, we can infer that the physiological variables that compose the POSSUM are more accurate as predictors than all the other analysed elements that could also predict bed reservation in ICU. Moreover, we may notice that the POSSUM variables age, serum hemoglobin and systolic blood pressure would be sufficient to predict properly the vacancy allocation in the ICU.
\end{abstract}

KEYWORDS: Medical oncology; Neoplasms/surgery; Intensive care; Risk assessment; General surgery; Risk factors; Intensive care units; Indicators of morbidity and mortality.

Prêmio Oswaldo Cruz 2012 - Área Cirúrgica, XXXI Congresso Médico Universitário da FMUSP - COMU 2012.

1. Acadêmica do $6^{\circ}$ do Curso de Medicina da Faculdade de Medicina da Universidade de São Paulo.

2. Divisão de Anestesiologia do Hospital das Clínicas, Professor Titular da Faculdade de Medicina da Universidade de São.

Endereço para correspondência: Av. Dr. Enéas de Carvalho Aguiar, 155 - $8^{\circ}$ andar - Prédio dos Ambulatórios - Bloco 3. CEP: 05403 000, São Paulo, SP. E-mail: tatiana.kronemberger@gmail.com 


\section{INTRODUÇÃO}

Tendo por objetivo a predição da morbi-mortalidade de pacientes submetidos a cirurgias e que teriam a necessidade de utilizarem a Unidade de Terapia Intensiva no período pós-operatório imediato, vários escores têm sido testados e passaram a ser utilizados ao longo do tempo pelas equipes cirúrgicas nos hospitais.

Além da necessidade de avaliar o risco cirúrgico inerente ao procedimento para cada paciente individualmente, o aprimoramento dos escores de predição de risco servem como importante ferramenta para melhoria do gerenciamento de recursos humanos, financeiros e estruturais, bem como, para assegurar a qualidade de atendimento hospitalar ao paciente submetido às cirurgias eletivas.

Um dos problemas ainda não equacionados principalmente nos hospitais públicos é o número de vagas de UTI que nem sempre encontram-se ajustados à demanda das cirurgias eletivas, o que suscita muitas vezes cancelamentos de última hora ou adiamentos dos procedimentos cirúrgicos. A reserva de vagas em Unidades de Terapia Intensiva é feita muitas vezes de maneira subjetiva, levando-se em conta a experiência da equipe cirúrgica e o estado de saúde do paciente no período prévio de acompanhamento em ambulatório. Isto pode ser comprovado pelos dados da instituição onde foi desenvolvido este projeto, consolidados entre abril 2010 a dezembro 2010 (Tabela 1), quando no período selecionado foram realizadas 4.461 cirurgias, sendo reservados 557 leitos de UTI para o pós-operatório imediato (POI). Destes, apenas 337 leitos foram efetivamente utilizados, o que representa $60,50 \%$ dos leitos previamente reservados. Merecem destaque os 129 leitos de UTI que foram utilizados no POI de cirurgias eletivas nas quais não havia sido prevista a necessidade de reserva de leito de UTI.

Em vista disso, se faz necessário o estudo sobre a previsão adequada da necessidade de UTI pós-operatória, bem como de um instrumento que faça a predição da morbi-mortalidade com maior exatidão e a indicação mais precisa de UTI, constituindo um dos mecanismos para diminuir possíveis complicações que possam advir do procedimento a que o paciente foi exposto.

Este estudo foi realizado em hospital terciário especializado no tratamento de neoplasias. Conforme pode ser verificado no Gráfico 1, houve um grande volume de cirurgias oncológicas no período de janeiro de 2009 a setembro de 2011.

O Gráfico 2 ilustra a taxa de ocupação de leitos de UTI da instituição no período de outubro de 2010 a setembro de 2011.

Existem vários escores na literatura para predizer morbi-mortalidade e necessidade de UTI, como nas cirurgias não cardíacas, por exemplo, o índice de Goldman, proposto em 1970, no qual os autores estabeleceram nove fatores de risco independentes para estimar o risco cardíaco. As variáveis estudadas foram as seguintes: idade, presença de infarto nos últimos seis meses, presença de estase jugular e/ou terceira bulha cardíaca, estenose aórtica importante, ritmo não sinusal, registro de extra-sístole ventricular freqüente $>5 / \mathrm{min}$, estado clínico precário observado a partir de dados laboratoriais, tipo de cirurgia e cirurgia de emergência ${ }^{1}$. Detsky et al. ${ }^{2}$, em 1986, modificou o índice proposto por Goldman ao acrescentar novas variáveis e atribuir pontuações diferentes para variáveis preexistentes, constituindo um dos índices de avaliação de risco perioperatório mais utilizado até os dias atuais. Porém, não foi estabelecido um escore para avaliação de risco cirúrgico específico para a população oncológica.

Tabela 1. Dados consolidados da instituição no período de abril de 2010 a dezembro de 2010

\begin{tabular}{|c|c|c|c|c|c|c|}
\hline \multicolumn{7}{|c|}{ Pós operatório imediato } \\
\hline & $\begin{array}{l}\text { Produção } \\
\text { cirúrgica - } \\
2010\end{array}$ & $\begin{array}{l}\text { Reservas } \\
\text { UTI } 2010\end{array}$ & $\begin{array}{l}\text { Não reservadas e que } \\
\text { utilizaram UTI - } 2010\end{array}$ & $\begin{array}{l}\text { Reservadas que } \\
\text { foram utilizadas }\end{array}$ & $\begin{array}{l}\text { Média de per- } \\
\text { manência UTI } \\
\text { cirúrgico }\end{array}$ & $\begin{array}{l}\text { Média de } \\
\text { permanência } \\
\text { cirúrgico }\end{array}$ \\
\hline Abril & 431 & 65 & 22 & 34 & 7,14 & 4,39 \\
\hline Maio & 461 & 66 & 21 & 41 & 6,57 & 3,93 \\
\hline Junho & 459 & 56 & 20 & 33 & 6,83 & 4,66 \\
\hline Julho & 503 & 58 & 13 & 42 & 8,19 & 4,19 \\
\hline Agosto & 543 & 74 & 13 & 53 & 5,22 & 4,11 \\
\hline Setembro & 509 & 66 & 13 & 42 & 5,01 & 4,47 \\
\hline Outubro & 559 & 56 & 10 & 33 & 4,00 & 4,22 \\
\hline Novembro & 508 & 61 & 9 & 34 & 3,00 & 4,65 \\
\hline Dezembro & 488 & 55 & 8 & 25 & 4,66 & 4,61 \\
\hline Total & 4461 & 557 & 129 & 337 & 5,62 & 4,36 \\
\hline
\end{tabular}


Gráfico 1. Tratamento de neoplasias oncológicas referente ao volume de cirurgias no período de janeiro de 2009 a outubro de 2011

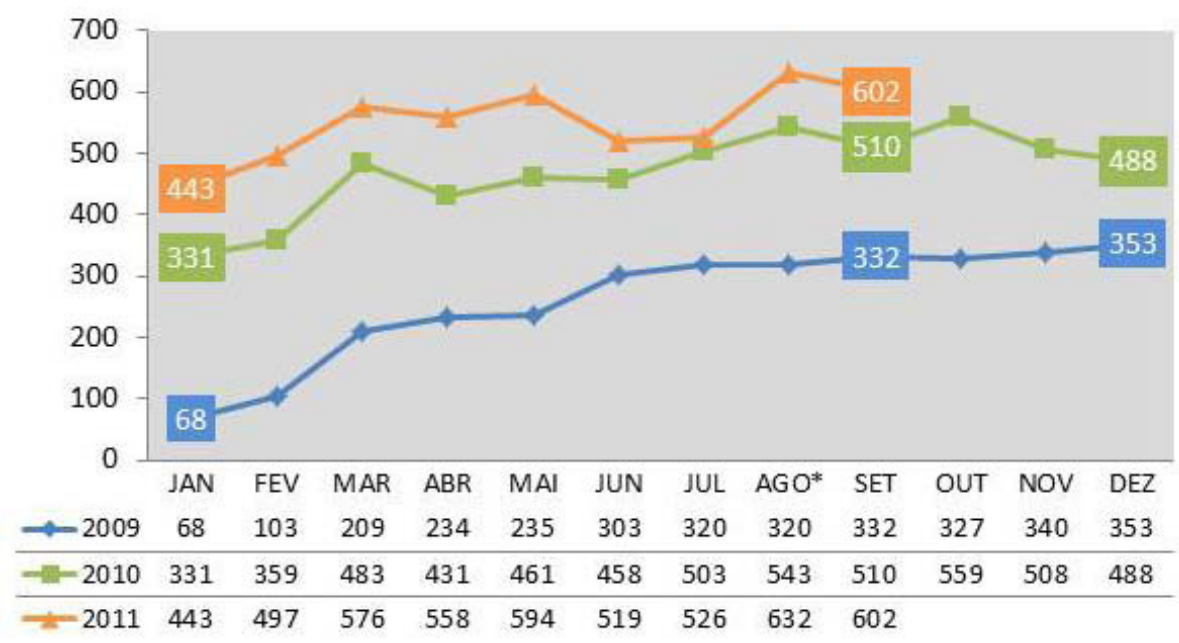

A American Society of Anesthesiology (ASA), no início da década de 60 , propôs o primeiro instrumento para avaliar universalmente o risco cirúrgico. No entanto, os parâmetros são subjetivos, permanecendo sujeitos à variabilidade de interpretação, além de levar em conta apenas fatores inerentes ao paciente, não considerando o trauma cirúrgico ${ }^{3}$.

O escore POSSUM ${ }^{5}$ (Physiological and Operative Severity Score for the enumeration of mortality and morbidity) foi descrito pela primeira vez por Copeland et $\mathrm{al}^{4}{ }^{\mathrm{e}}$ em 1991. O risco relativo referente à morbidade/ mortalidade desse escore é dado por um modelo exponencial formado pela soma de 12 variáveis fisiológicas e seis cirúrgicas; de acordo com a gravidade do paciente e a complexidade da cirurgia ${ }^{4,5}$. Dentre as variáveis fisiológicas estão: idade, história prévia de cardiopatia, história prévia de pneumopatia, pressão arterial, freqüência cardíaca, escala de coma de Glasgow, níveis de hemoglobina, número de leucócitos, níveis séricos de uréia, sódio e potássio, além de alterações no eletrocardiograma. As variáveis perioperatórias incluem: complexidade cirúrgica, número de procedimentos, perda sanguínea, contaminação peritoneal, existência de metástase e tipo de cirurgia (eletiva, urgência ou emergência) ${ }^{4}$.

Gráfico 2. Taxa de ocupação de leitos de UTI, outubro de 2010 a setembro 2011

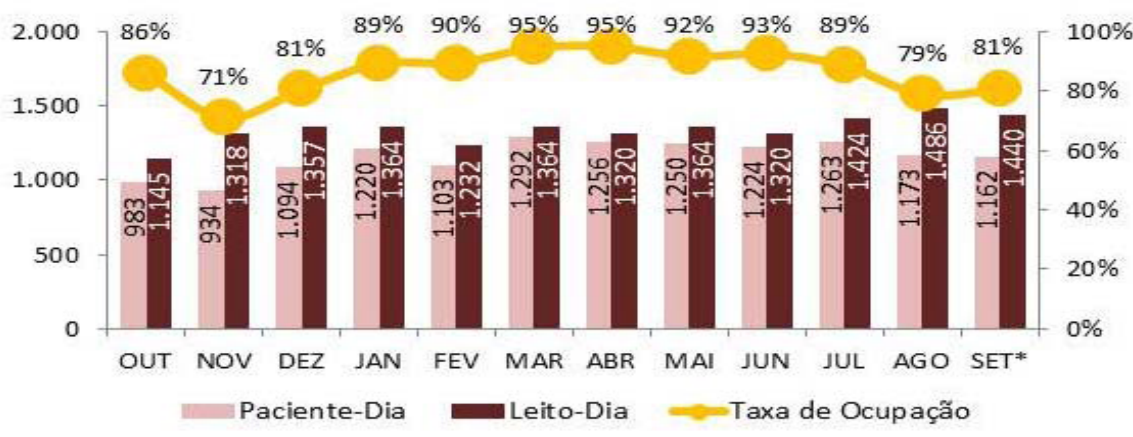

O escore POSSUM permite não só a predição da morbi-mortalidade nos pacientes cirúrgicos, como também comparar o desempenho de diferentes equipes cirúrgicas ou unidades hospitalares através da estratificação dos pacientes ${ }^{6,7}$. Pode também auxiliar a identificar se a morbidade/mortalidade está mais relacionada com a gravidade dos pacientes ou a complexidade das cirurgias realizadas ${ }^{5,8}$. 
Outro índice estudado foi a escala de performance status de Karnofsky, que classifica os pacientes de acordo com o grau de deficiências funcionais ocasionadas pela enfermidade que os acomete. Este índice pode ser utilizado para determinação da possibilidade de receber quimioterapia, da necessidade de ajuste das doses destas medicações, bem como avaliar o bem estar do paciente frente ao tratamento a que será submetido, sendo clínico ou cirúrgico. É um instrumento que conta com a avaliação feita apenas pelo médico.

O aprimoramento de sistemas de predição de risco com estratificação dos diversos tipos de população e redução das interferências externas é uma importante ferramenta para auxiliar no gerenciamento de recursos humanos, financeiros e estruturais, assim como para assegurar qualidade de atendimento. Atualmente a previsão de suporte intensivo pósoperatório para cirurgias eletivas é estabelecida de maneira subjetiva, podendo inclusive comprometer o desempenho do hospital levando à suspensão de cirurgias por falta de vagas de UTI. Existem escalas propostas para predizer o tempo de internação em terapia intensiva de algumas cirurgias que regularmente necessitam de suporte intensivo pósoperatório, como por exemplo, cirurgia cardíaca. No entanto, muito pouco se discute sobre a possibilidade de previsão da necessidade de UTI pós-operatória. Possivelmente, da mesma maneira que o escore POSSUM considera variáveis fisiológicas e cirúrgicas, a predição da necessidade de suporte intensivo pós-operatório também deverá contemplar ambas variáveis. Atualmente, em muitos casos, ocorre a solicitação rotineira de reserva de leitos intensivos para pós-operatório de pacientes muito graves (ASA III e ASA IV) que são submetidos a procedimentos cirúrgicos de pequeno porte, que não necessitariam do leito, o qual permanece bloqueado por um determinado período, não sendo utilizado. O desenvolvimento de uma escala preditiva de simples utilização pode minimizar estes transtornos e otimizar o gerenciamento interno de leitos cirúrgicos e de terapia intensiva.

\section{OBJETIVO}

Quando propusemos este estudo em pacientes cirúrgicos oncológicos nosso objetivo foi identificar a partir do POSSUM um escore que pudesse predizer adequadamente os resultados cirúrgicos de curto prazo (até 30 dias no pósoperatório), para a necessidade de suporte intensivo pósoperatório. Também procurou-se correlacionar o POSSUM, índice pouco conhecido e pouco utilizado no nosso meio, com outros índices como o ASA e Karnofsky.

\section{MÉTODOS PARA PRIMEIRA FASE DO ESTUDO}

Na primeira fase deste estudo, utilizamos uma amostra seqüencial de 61 pacientes, que demonstrou baixo índice de complicações e internações na UTI ${ }^{9}$.

Utilizamos naquela ocasião o teste exato de Mann Whitney para comparar as medianas das populações que foram internadas na UTI e as que não foram internadas segundo as Escalas ASA e POSSUM e o Índice de Karnofsky (Tabela 2).

Tabela 2. Mediana das populações

\begin{tabular}{l|c|c|c|c}
\hline Variável & UTI & Mediana & $\mathrm{N}$ & p-valor \\
\hline \multirow{2}{*}{ ASA } & Não & 2 & 55 & \multirow{2}{*}{0,1932} \\
\cline { 2 - 4 } & Sim & 2 & 6 & \\
\hline \multirow{2}{*}{ Karnofsky } & Não & 1 & 55 & \multirow{2}{*}{0,0746} \\
\cline { 2 - 4 } & Sim & 0,85 & 6 & \\
\hline \multirow{2}{*}{ POSSUM } & Não & 0,15 & 55 & \multirow{2}{*}{0,0050} \\
\cline { 2 - 4 } & Sim & 0,36 & 6 & \\
\hline
\end{tabular}

Pelo resultado do teste, para esse estudo, observamos que não existiu diferença entre as medianas da Escala ASA e do Índice de Karnofsky, ou seja, a Escala ASA e o Índice de Karnofsky não discriminavam a população que deveria ser internada na UTI. O mesmo não ocorreu com a Escala POSSUM, que apresentou diferença estatisticamente significativa entre as medianas das duas amostras, ou seja, a Escala POSSUM, neste estudo e para tal amostra discriminou a opção de internar ou não um paciente na $\mathrm{UTI}^{9,10}$.

Dessa forma, a partir de dados mais próximos à realidade, procedemos ao cálculo amostral a fim de dar prosseguimento ao projeto, diminuindo-se para 9,8\% o percentual dos pacientes internados em UTI, em vez de uma média projetada ao redor de 13\% (média histórica da instituição). Assim, para confirmar neste estudo que o POSSUM possa prever a internação em UTI, o número mínimo passaria a ser de 136 pacientes a serem seguidos. Para este cálculo foi considerada uma variação de $5 \%$, erro alfa de $5 \%$, poder de $95 \%$ e perda de aproximadamente $5 \%$ quanto ao seguimento dos pacientes.

Dessa maneira, para que houvesse um número substancial, analisamos 186 prontuários de pacientes submetidos a cirurgias oncológicas na instituição onde se desenvolveu este projeto, que apresentaram todas as informações completas, de modo que o estudo fosse o mais fidedigno possível. Assim, apresentamos os dados referentes a reservas de leito em UTI, sua real utilização, bem como os dados referentes aos escores ASA, Karnofsky e os parâmetros fisiológicos do POSSUM, para que pudessem ser comparados.

\section{Métodos para segunda fase do estudo}

Este estudo completo apresenta a análise dos 
prontuários de 186 pacientes com câncer de qualquer natureza e que foram submetidos a cirurgias oncológicas na instituição onde se desenvolveu o projeto, sendo 90 mulheres $(45,9 \%)$ e 96 homens $(54,1 \%)$.

Gráfico 3. Faixas etárias da população da amostra

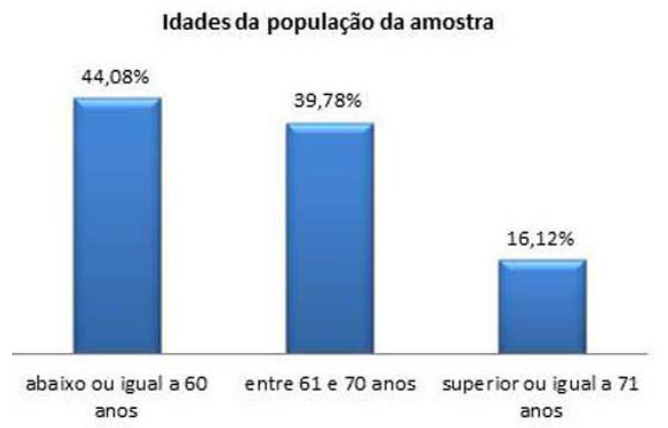

Os pacientes foram avaliados conforme os critérios ASA, Karnofsky e as diversas características fisiológicas que compõem o escore de POSSUM e criado a somatória da parte fisiológica deste escore.

Para responder as perguntas deste estudo, primeiramente foram descritos os pacientes segundo reserva e utilização da UTI com uso de frequências absolutas e relativas.

\section{RESULTADOS}

Os resultados de cada um das variáveis foram descritos segundo utilização dos leitos de terapia intensiva com uso de medidas resumo (frequências absolutas e relativas ou medianas, mínimo e máximo) e comparados os critérios segundo utilização da UTI com uso de testes Mann-Whitney ${ }^{10}$. Foram criadas as curvas ROC (Receiver Operating Characteristic $)^{11}$ dos mesmos e calculadas as áreas sob cada uma das curvas para verificar quais apresentam melhor predição da utilização do leito de terapia intensiva.

Cada uma das variáveis que compõem a parte fisiológica do POSSUM foi descrita segundo utilização real das vagas de UTI. Também verificou-se a existência de associação destas variáveis com a utilização da UTI com uso de testes qui-quadrado ${ }^{10}$ ou testes exatos de Fisher ou testes da razão de verossimilhanças quando a amostra foi insuficiente para aplicação do teste qui-quadrado ${ }^{10}$. Esses testes consideram as categorias de cada uma das variáveis de maneira nominal. Foi aplicado, também, teste qui-quadrado de tendência ${ }^{10}$, que considera a ordenação atribuída para cada uma das variáveis fisiológicas para verificar se a utilização das ponderações são consistentes à
As faixas etárias da população que compõe a amostra, bem como as localizações das neoplasias, estão descritas nos Gráficos 3 e 4.

Gráfico 4. Localização da neoplasia

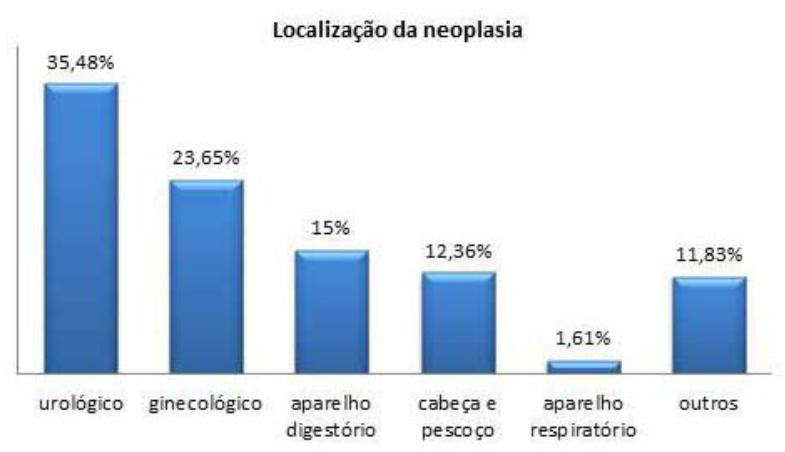

criação de um novo escore composto apenas por variáveis obtidas no período pré-operatório.

Foi criado um modelo de regressão logística ${ }^{12}$ usando a soma das variáveis fisiológicas do POSSUM e outro modelo apenas com as variáveis que se apresentaram importantes na predição da utilização das vagas de UTI, a fim de compará-los. Para cada um dos modelos criados, foram calculadas a sensibilidade, a especificidade, valor preditivo positivo (VPP), valor preditivo negativo (VPN) e a área sob a curva ROC fornecida pelas probabilidades preditas de utilização do leito de UTI segundo cada modelo. de $5 \%$.

Os testes foram realizados com nível de significância

A Tabela 3 mostra que quase metade dos leitos reservados não foram utilizados efetivamente.

Tabela 3. Descrição da reserva de UTI e utilização de UTI nos pacientes do estudo

\begin{tabular}{c|c|c}
\hline Variável & Frequência & $\%$ \\
\hline Reserva de UTI & & \\
\hline Não & 132 & 71,0 \\
\hline Sim & 54 & 29,0 \\
\hline Utilizou UTI & & \\
\hline Não & 155 & 83,3 \\
\hline Sim & 31 & 16,7 \\
\hline Total & 186 & 100 \\
\hline
\end{tabular}

A Tabela 4 mostra que todos os métodos apresentam diferenças estatisticamente significativas entre pacientes que utilizam e que não utilizam a UTI $(\mathrm{p}<0,05)$. 
Tabela 4. Descrição dos critérios ASA, Karnofsky e soma da parte fisiológica do POSSUM segundo utilização da UTI e resultado do teste comparativo

\begin{tabular}{|c|c|c|c|c|c|c|}
\hline \multirow{3}{*}{ Variável } & \multicolumn{4}{|c|}{ Utilizou UTI } & \multirow{3}{*}{ Total } & \multirow{3}{*}{$\mathrm{p}$} \\
\hline & \multicolumn{2}{|c|}{ Não } & \multicolumn{2}{|c|}{ Sim } & & \\
\hline & $\mathrm{n}$ & $\%$ & $\mathrm{n}$ & $\%$ & & \\
\hline$\overline{\mathrm{ASA}}$ & & & & & & 0,006 \\
\hline I & 24 & 88,9 & 3 & 11,1 & 27 & \\
\hline II & 113 & 86,9 & 17 & 13,1 & 130 & \\
\hline III & 18 & 62,1 & 11 & 37,9 & 29 & \\
\hline KARNOFSKY & \multirow{2}{*}{\multicolumn{2}{|c|}{$90(30,100)$}} & \multirow{2}{*}{\multicolumn{2}{|c|}{$80(30,100)$}} & & $<0,001$ \\
\hline $\begin{array}{l}\text { Mediana (mínimo, máximo } \\
\text { POSSUM }\end{array}$ & & & & & $90(30,100)$ & \\
\hline Mediana (mínimo, máximo & \multicolumn{2}{|c|}{$14(12,28)$} & \multicolumn{2}{|c|}{$17(12,36)$} & $14,5(12,36)$ & $<0,001$ \\
\hline
\end{tabular}

Resultado do teste Mann-Whitney

Gráfico 5. Curva ROC do critério ASA para predição de utilização de UTI

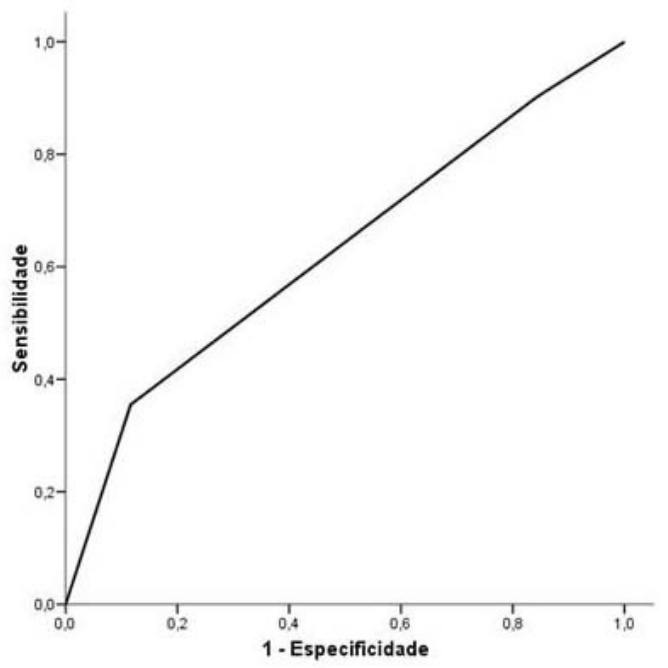

Gráfico 6. Curva ROC do critério KARNOFSKY para predição de utilização de UTI

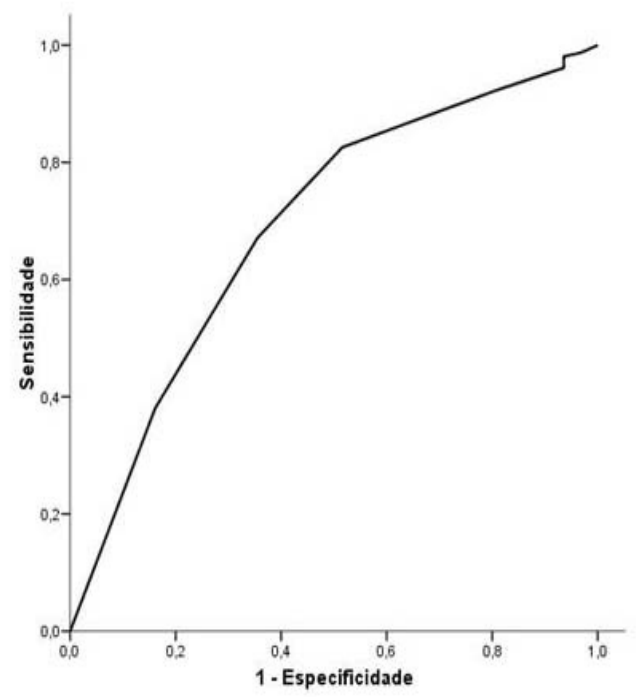

Gráfico 7. Curva ROC do critério POSSUM (fisiológico) para predição de utilização de UTI

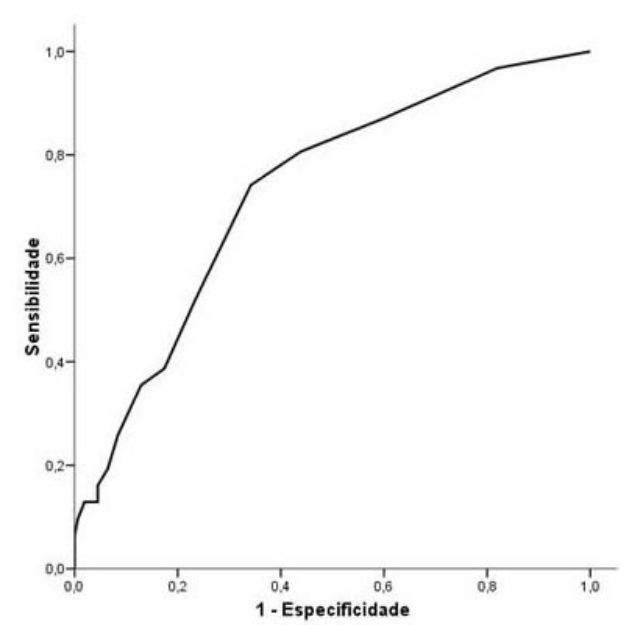

Tabela 5. Valores das áreas sob a curva ROC segundo cada critério

\begin{tabular}{|c|c|c|c|c|}
\hline \multirow{2}{*}{ Critério } & \multirow{2}{*}{$\begin{array}{c}\text { Área } \\
\text { (ROC) }\end{array}$} & \multirow{2}{*}{$\begin{array}{c}\text { Erro } \\
\text { padrão }\end{array}$} & \multicolumn{2}{|c|}{ IC (955) } \\
\hline & & & Inferior & Superior \\
\hline ASA & 0,627 & 0,059 & 0,511 & 0,742 \\
\hline Karnofsky & 0,692 & 0,053 & 0,588 & 0,796 \\
\hline $\begin{array}{l}\text { POSSUM } \\
\text { (fisiológico) }\end{array}$ & 0,729 & 0,048 & 0,635 & 0,822 \\
\hline
\end{tabular}

Dentre os gráficos obtidos, podemos observar que o Gráfico 7 possui a maior área sob a curva, sugerindo melhor predição da utilização de UTI pela soma das partes fisiológicas do POSSUM, porém os intervalos de confiança mostram que as áreas sob a curva ROC são próximas e estatisticamente iguais, pois os intervalos se sobrepõem. 
Tabela 6. Descrição de cada uma das variáveis fisiológicas individuais segundo uso da UTI

\begin{tabular}{|c|c|c|c|c|c|c|c|}
\hline \multirow{3}{*}{ Variável } & \multicolumn{4}{|c|}{ Utilizou UTI } & \multirow{3}{*}{ Total } & \multirow{3}{*}{$\mathbf{p}$} & \multirow{3}{*}{$\begin{array}{l}\text { p Teste de } \\
\text { tendência }\end{array}$} \\
\hline & \multicolumn{2}{|c|}{ Não } & \multicolumn{2}{|c|}{ Sim } & & & \\
\hline & $\mathrm{n}$ & $\%$ & $\mathrm{n}$ & $\%$ & & & \\
\hline Idade & & & & & & $0,063 \#$ & 0,274 \\
\hline$<60$ & 88 & 88,9 & 11 & 11,1 & 99 & & \\
\hline 60 a 70 & 47 & 74,6 & 16 & 25,4 & 63 & & \\
\hline$>70$ & 20 & 83,3 & 4 & 16,7 & 24 & & \\
\hline Doença cardíaca & & & & & & $0,509 \#$ & 0,706 \\
\hline Ausente & 104 & 85,2 & 18 & 14,8 & 122 & & \\
\hline Uso de medicamentos & 44 & 78,6 & 12 & 21,4 & 56 & & \\
\hline Edema ou dispnéia & 3 & 100,0 & 0 & 0,0 & 3 & & \\
\hline Turgência de jugular, cardiomegalia, B3 & 4 & 80,0 & 1 & 20,0 & 5 & & \\
\hline Doença respiratória & & & & & & $0,710 \#$ & 0,515 \\
\hline Ausente & 120 & 84,5 & 22 & 15,5 & 142 & & \\
\hline DPOC leve & 26 & 81,3 & 6 & 18,8 & 32 & & \\
\hline DPOC moderada & 8 & 72,7 & 3 & 27,3 & 11 & & \\
\hline Dispnéia em repouso, DPOC grave & 1 & 100,0 & 0 & 0,0 & 1 & & \\
\hline PAS & & & & & & $0,065 \#$ & 0,010 \\
\hline 110 a 129 & 91 & 88,3 & 12 & 11,7 & 103 & & \\
\hline 100 a 109 ou 130 a 170 & 55 & 78,6 & 15 & 21,4 & 70 & & \\
\hline 90 a 99 ou $>170$ & 9 & 75,0 & 3 & 25,0 & 12 & & \\
\hline$<90$ & 0 & 0,0 & 1 & 100,0 & 1 & & \\
\hline FC & & & & & & $0,291 \#$ & 0,190 \\
\hline 50 a 80 & 135 & 83,9 & 26 & 16,1 & 161 & & \\
\hline 40 a 49 ou 81 a 100 & 18 & 85,7 & 3 & 14,3 & 21 & & \\
\hline 101 a 120 & 2 & 50,0 & 2 & 50,0 & 4 & & \\
\hline Glasgow & & & & & & $0,167^{*}$ & 0,025 \\
\hline 15 & 155 & 83,8 & 30 & 16,2 & 185 & & \\
\hline 12 a 14 & 0 & 0,0 & 1 & 100,0 & 1 & & \\
\hline Uréia & & & & & & $0,149 \#$ & 0,047 \\
\hline$<45,18$ & 144 & 84,2 & 27 & 15,8 & 171 & & \\
\hline 45,18 a 60,84 & 10 & 83,3 & 2 & 16,7 & 12 & & \\
\hline 60,84 a 90,36 & 1 & 33,3 & 2 & 66,7 & 3 & & \\
\hline Potássio & & & & & & $0,088 \#$ & 0,016 \\
\hline 3,5 a 5,0 & 148 & 84,6 & 27 & 15,4 & 175 & & \\
\hline 3,1 a 3,4 ou 5,1 a 5,3 & 7 & 70,0 & 3 & 30,0 & 10 & & \\
\hline 2,9 a 3,1 ou 5,4 a 5,9 & 0 & 0,0 & 1 & 100,0 & 1 & & \\
\hline Sódio & & & & & & $0,148 \#$ & 0,034 \\
\hline$>136$ & 152 & 84,0 & 29 & 16,0 & 181 & & \\
\hline 131 a 135 & 3 & 75,0 & 1 & 25,0 & 4 & & \\
\hline 126 a 130 & 0 & 0,0 & 1 & 100,0 & 1 & & \\
\hline $\mathrm{Hb}(\mathrm{g} / \mathrm{l})$ & & & & & & $<0,001 \#$ & $<0,001$ \\
\hline 13 a 16 & 120 & 90,2 & 13 & 9,8 & 133 & & \\
\hline 11,5 a 12,9 ou 16,1 a 17,0 & 28 & 73,7 & 10 & 26,3 & 38 & & \\
\hline 10,0 a 11,4 ou 17,1 a 18,0 & 5 & 45,5 & 6 & 54,5 & 11 & & \\
\hline$<10$ ou $>18$ & 2 & 50,0 & 2 & 50,0 & 4 & & \\
\hline Leucócitos & & & & & & $0,060 \#$ & 0,054 \\
\hline 4.000 a 10.000 & 149 & 85,1 & 26 & 14,9 & 175 & & \\
\hline 10.100 a 20.000 ou 3.100 a 3.900 & 4 & 50,0 & 4 & 50,0 & 8 & & \\
\hline$>20.000$ ou $<3.100$ & 2 & 66,7 & 1 & 33,3 & 3 & & \\
\hline ECG & & & & & & $0,096 \#$ & 0,006 \\
\hline Normal & 144 & 85,2 & 25 & 14,8 & 169 & & \\
\hline BRE, RDAS, BRD & 6 & 85,7 & 1 & 14,3 & 7 & & \\
\hline FA & 1 & 50,0 & 1 & 50,0 & 2 & & \\
\hline Sinal de IAM antigo & 4 & 50,0 & 4 & 50,0 & 8 & & \\
\hline Total & 155 & 83,3 & 31 & 16,7 & 186 & & \\
\hline
\end{tabular}

A Tabela 7, através dos resultados de associação e dos testes de tendência, mostra que apenas a medida de $\mathrm{Hb}$ é estatisticamente associada à utilização de UTI $(\mathrm{p}<0,001)$. Os percentuais observados nos pacientes do estudo sugerem aumento percentual de utilização da UTI conforme aumento das categorias de cada uma das variáveis que compõem a parte fisiológica do POSSUM, logo a utilização do teste de tendência se mostra bem aplicada e tem-se que PAS, escala de nível de consciência (Glasgow), níveis séricos de uréia, potássio e sódio, Hb e alterações no ECG apresentam estatisticamente aumento do percentual de utilização de UTI conforme aumento da pontuação destas variáveis $(\mathrm{p}<0,05)$. 
Tabela 7. Resultado dos modelos de predição de utilização de UTI segundo variáveis importantes e segundo soma dos critérios fisiológicos do POSSUM

\begin{tabular}{|c|c|c|c|c|c|c|c|c|c|}
\hline \multirow{2}{*}{ Modelo } & \multirow{2}{*}{ Fator } & \multirow{2}{*}{ Coeficiente } & \multirow{2}{*}{$\begin{array}{c}\text { Erro } \\
\text { Padrão }\end{array}$} & \multirow{2}{*}{$\begin{array}{c}\text { Estatística } \\
\text { de Wald }\end{array}$} & \multirow{2}{*}{ gl } & \multirow{2}{*}{ OR } & \multicolumn{2}{|c|}{ IC $(95 \%)$} & \multirow{2}{*}{$\mathbf{p}$} \\
\hline & & & & & & & Inferior & Superior & \\
\hline \multirow{11}{*}{$\begin{array}{l}\text { Uso das } \\
\text { categorias }\end{array}$} & Idade (ref. < 60) & & & & & & & & \\
\hline & 60 a 70 & 1,019 & 0,489 & 4,334 & 1 & 2,77 & 1,06 & 7,22 & 0,037 \\
\hline & $>70$ & $-0,0091$ & 0,72 & 0,000 & 1 & 0,99 & 0,24 & 4,04 & 0,990 \\
\hline & $\mathrm{Hb}(\mathrm{g} / \mathrm{l})($ ref. 13 a 16$)$ & & & & & & & & \\
\hline & 11,5 a 12,9 ou 16,1 a 17,0 & 1,65 & 0,53 & 9,61 & 1 & 5,19 & 1,83 & 14,72 & 0,002 \\
\hline & 10,0 a 11,4 ou 17,1 a 18,0 & 2,79 & 0,75 & 13,92 & 1 & 16,34 & 3,77 & 70,88 & $<0,001$ \\
\hline & $<10$ ou $>18$ & 2,49 & 1,20 & 4,32 & 1 & 12,08 & 1,15 & 126,46 & 0,038 \\
\hline & PAS (ref. 110 a 129) & & & & & & & & \\
\hline & 100 a 109 ou 130 a 170 & 0,92 & 0,51 & 3,31 & 1 & 2,52 & 0,93 & 6,82 & 0,069 \\
\hline & $<99$ ou $>170$ & 1,67 & 0,78 & 4,62 & 1 & 5,30 & 1,16 & 24,21 & 0,032 \\
\hline & Constante & $-3,35$ & 0,52 & 42,04 & 1 & & & & $<0,001$ \\
\hline \multirow{2}{*}{$\begin{array}{l}\text { POSSUM } \\
\text { (fisiológico) }\end{array}$} & POSSUM & 0,17 & 0,05 & 13,63 & 1 & 1,19 & 1,08 & 1,30 & $<0,001$ \\
\hline & Constante & $-4,46$ & 0,82 & 29,58 & 1 & & & & $<0,001$ \\
\hline
\end{tabular}

A Tabela 8 mostra que apenas as variáveis idade, níveis séricos de Hb e PAS são estatisticamente suficientes para predizer a utilização de UTI $(\mathrm{p}<0,05)$ nos pacientes avaliados. A soma das partes fisiológicas do POSSUM apresentam aumento da chance de uso da UTI em 19\% $(\mathrm{OR}=1,19)$ a cada aumento de 1 ponto na soma, sendo a probabilidade de predição da utilização da UTI com a soma das partes fisiológicas do POSSUM calculadas com a fórmula abaixo:

$\operatorname{Prob}(\mathrm{UTI})=\frac{\exp (-4,46+0,17 \mathrm{xsoma} \text { fisiológica }(\text { POSSUM }))}{1+\exp (-4,46+0,17 \text { xsoma fisiológica }(\text { POSSUM }))}$

Portanto, podemos observar que três dentre as doze variáveis fisiológicas que compõem o POSSUM, nesta amostra, são suficientes para predizer a necessidade da reserva de vaga na unidade de terapia intensiva.

Tabela 8. Medidas de avaliação dos modelos

\begin{tabular}{c|l|c|c|c}
\hline \multirow{2}{*}{ Modelo } & \multirow{2}{*}{ Medida } & \multirow{2}{*}{ Estimativa } & \multicolumn{2}{|c}{ IC (95\%) } \\
\cline { 4 - 5 } & & & Inferior & Superior \\
\hline \multirow{4}{*}{ Uso das categorias } & Sensibilidade & 19,49 & $7,5 \%$ & $37,5 \%$ \\
& Especificidade & $96,8 \%$ & $92,6 \%$ & $98,9 \%$ \\
& Área (ROC) & 0,581 & 0,509 & 0,653 \\
& VPP & $54,5 \%$ & $23,4 \%$ & $83,3 \%$ \\
& VPN & $85,7 \%$ & $79,6 \%$ & $90,5 \%$ \\
\hline \multirow{5}{*}{ POSUM (fisiológico) } & Sensibilidade & $12,9 \%$ & $3,6 \%$ & $29,8 \%$ \\
& Área (ROC) & $9,19 \%$ & $94,4 \%$ & $99,6 \%$ \\
& VPP & $57,1 \%$ & 0,494 & 0,616 \\
& VPN & $84,9 \%$ & $18,4 \%$ & $90,1 \%$ \\
& & & $78,8 \%$ & $89,8 \%$ \\
\hline
\end{tabular}

A Tabela 8 mostra que os modelos apresentam resultados bastante semelhantes, sendo o modelo com uso das categorias nominais um pouco mais sensível e menos específico que o modelo com a soma da parte fisiológica do POSSUM.

\section{CONCLUSÃO}

Na primeira fase deste estudo, considerando-se uma população de pacientes com diagnóstico oncológico variável, obtida a partir dos agendamentos ambulatoriais na instituição, permitiu-se inferir que apenas o POSSUM, dentre os índices utilizados neste trabalho, pode predizer a internação na UTI.

Também podemos considerar que, como o POSSUM acrescenta em sua fórmula variáveis intraoperatorias, pode-se questionar sua utilização rotineira como preditor de necessidade internação em UTI, daí a necessidade de buscarmos avaliar em separado as variáveis fisiológicas 
que compõem tal escore.

Como mencionado, os dados da instituição apontam uma imprecisão de quase $30 \%$ das indicações de UTI pela equipe cirúrgica. Este fato per si justifica a busca de um outro preditor, haja visto a subjetividade dos métodos atuais. Considerando a primeira fase do estudo, não conseguimos demonstrar que o ASA e Karnosfski possam ter significância estatística como preditores de necessidade de UTI, deste modo, optamos por ampliar o estudo do índice POSSUM, a fim de verificar quais fatores seriam mais precisos para a indicação de UTI dos pacientes submetidos a cirurgias oncológicas em nossa instituição. Entretanto, a partir de análise estatística mais aprofundada, questionamos se seria possível propor um novo escore utilizando apenas os fatores mais relevantes para a correta indicação de necessidade de UTI a partir dos dados da população que compõe este estudo, reduzindo-se o número de fatores a serem analisados pela equipe cirúrgica no momento da reserva de leitos de terapia intensiva para seus pacientes.

Essa abordagem pode mostrar se os dezoito itens que compõem o POSSUM têm a variabilidade esperada para diferenciar níveis de gravidade.

Assim, na segunda fase do estudo, procuramos correlacionar os índices ASA, Karnofsky e as variáveis fisiológicas do POSSUM, a partir dos dados da amostra, a fim de nos certificarmos qual seria o melhor preditor para a necessidade de UTI no período pós-operatório das cirurgias

\section{REFERÊNCIAS}

1. Fleischman KE, Goldman L, Young B, Lee T. Association between cardiac and noncardiac complications in patients undergoing noncardiac surgery: outcomes and effects on length of stay. AJM. 2003;115:515-20. http://dx.doi. org/10.1016/S0002-9343(03)00474-1

2. Detsky AS, Abrams HB, Forbath N, et al. Cardiac assessment for patients undergoing noncardiac surgery: a multifactorial clinical risk index. Arch Intern Med. 1986;146(11):2131-4. doi:10.1001/archinte.1986.00360230047007.

3. Haynes SR, Lawler PG. An assessment of the consistency of ASA physical status classification allocation. Anaesthesia. 1995;50(3):195-9.

4. Copeland GP, Jones D, Walters M. POSSUM: a scoring system for surgical audit. Br J Surg. 1991;78(3):355-60.

5. Prytherch DR, Whiteley MS, Higgins B, et al. POSSUM and Portsmouth POSSUM for predicting mortality. Br J Surg. 1998;85:1217-20. doi: 10.1046/j.1365-2168. 1998.00840.x

6. Elias ACGP, Matsuo T, Grion CMC, et al. POSSUM escore como preditor de mortalidade em pacientes cirúrgicos. Rev Esc Enferm USP. 2009;43(1):23-9. Disponível em: http:// www.revistas.usp.br/reeusp/article/view/40322/43210 oncológicas realizadas na instituição onde se desenvolveu o projeto. Através das curvas ROC podemos inferir que as variáveis fisiológicas que compõem o POSSUM são melhores preditores que os outros índices analisados para a reserva do leito de UTI. Também notamos que as variáveis idade, nível sérico de hemoglobina e a medida da pressão arterial sistólica seriam suficientes para predizer a real necessidade da utilização da vaga na unidade de terapia intensiva.

Cumpre-se ressaltar que, de uma maneira inédita, a divisão do POSSUM em suas variáveis pré-operatórias e intra-operatórias pode vir a ser interessante, e foi esta uma das propostas da segunda fase deste estudo.

Descartamos as variáveis intra-oparatórias, pois estas fogem de uma previsibilidade no momento da reserva do leito de UTI pela equipe. Já o mesmo não ocorre com as variáveis fisiológicas, que são obtidas em consultas ambulatoriais e avaliação pré-operatória.

Seria ousado, substituir um índice com o ASA, já consagrado, com críticas pela sua subjetividade inerente, mas este estudo pode apontar um caminho para a utilização de variáveis fisiológicas como um instrumento mais objetivo para a reserva de vagas de UTI e sua real utilização.

Temos claro que para validação de nosso modelo, ainda se requer maior número amostral e análise estatística mais complexa, além da participação de várias instituições.

7. Tamijmarane A, Bhati CS, Mirza DF, et al. Application of Portsmouth modification of physiological and operative severity scoring system for enumeration of morbidity and mortality (P-POSSUM) in pancreatic surgery. W J Surg Oncol. 2008;6:39. doi: 10.1186/1477-7819-6-39.

8. Yii MK, Ng KJ. Risk-adjusted surgical audit with the POSSUM scoring system in a developing country. Br J Surg. 2002; 89:110-3. doi: 10.1046/j.0007-1323.2001.01979.x

9. Kronemberger TB, Simões CM, Pose RA, Auler JOC. Estudo para predição de morbimortalidade em cirurgia oncológica e necessidade de UTI: estudo preliminar. In: $8^{\circ}$ Congresso Paulista de Anestesiologia, 2011, São Paulo. Suplemento especial. São Paulo: Associação Paulista de Medicina; 2011. v. 129, p.35.

10. Hanley JA, McNeil BJ. The meaning and use of area under the Receiver Operating Characteristics (ROC) curve. Radiology. 1982;143:29-36.

11. Hosmer DW, Lemeshow S. Applied logistic regression. 2nd ed. New York: Willey; 2000. p.230.

12. Kirkwood BR, Sterne JAC. Essential medical statistics. 2nd ed. Massachussets, USA: Blackwell Science; 2006. p.502. 